\title{
Home-monitoring reduces hospital stay for COVID-19 patients
}

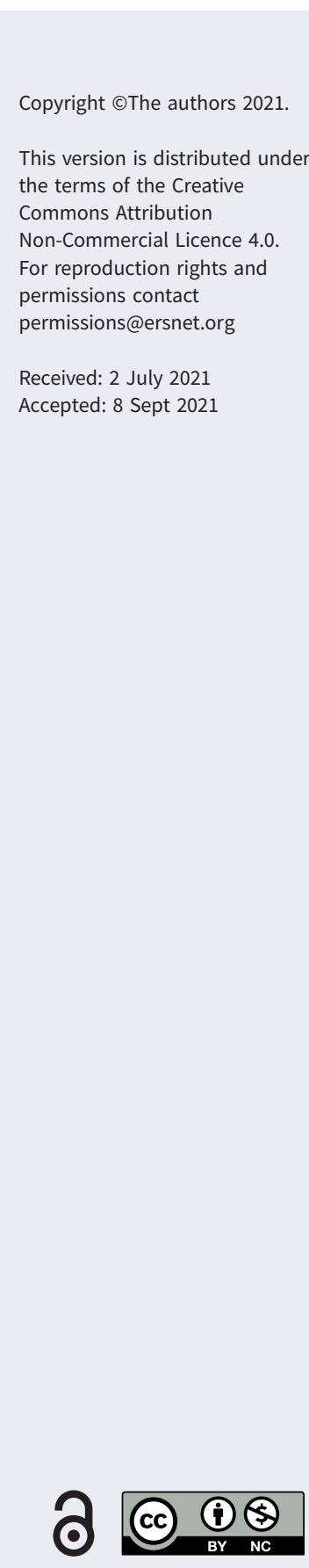

To the Editor:

With great interest we read the article by DiRIKGIL et al. [1], in which they demonstrate the potential of home monitoring to reduce hospital admissions by safely surveying clinical symptoms and vital parameters. They encourage the further consideration of strategies for home monitoring larger patient groups and, particularly, patients with confirmed coronavirus disease 2019 (COVID-19).

In March 2020, COVID-19 led to a pandemic. In April 2020 we rapidly developed and implemented home monitoring after hospital discharge in COVID-19 patients to reduce duration of hospital stay [2]. During the COVID-19 pandemic, home monitoring emerged as a new and powerful modality in several centres all around the world for various indications [1,3-6]. We would like to share our first-year experience with COVID-19 home monitoring in the largest patient cohort to date $(\mathrm{n}=320)$.

We retrospectively report on our experience with home monitoring for COVID-19 patients in the Netherlands. Patients hospitalised for COVID-19 with improving clinical trend and oxygen therapy tapered down to a maximum of $3 \mathrm{~L} \cdot \mathrm{min}^{-1}$, were approached for earlier discharge with home monitoring. Home monitoring consisted of twice daily control of oxygen saturation, temperature and symptoms. Warnings for deterioration or comments were sent directly to the home-monitoring team of medical residents supervised by pulmonologists. The team contacted patients by phone to assist in titration of oxygen, control clinical symptoms, and advise presentation to the hospital or general practitioner. Incidentally the team gave advice on the use of antimicrobials, antitussives, or the use of inhalers. A more detailed method description is available in Grutters et al. 2020 [2]. Main outcomes were reducing length of hospitalisation, safety, defining risk factors for readmission, and patient satisfaction.

We included all COVID-19 patients discharged with home monitoring from the St Antonius Hospital between 8 April, 2020 and 17 May, 2021. We recorded demographic data, diagnostic methods, admission to intensive care unit, oxygen therapy, fever at discharge, disease duration, length of hospitalisation and underlying comorbidities [7]. Reduction in length of hospitalisation was the sum of days receiving oxygen therapy at home plus one day, comparable to the hospital's ward protocol in which patients were discharged one day after the oxygen was tapered down. A maximum of 14 days was noted. We recorded duration of monitoring and number of telephone contacts. Furthermore, patients completed a satisfaction questionnaire based on Consumer Quality Index in General Practice questionnaires [8]. The united medical research ethics committees of the St Antonius Hospital approved this study (reference number W20.107/ Z20.065). Fisher's exact test for categorical data was used to define risk factors for readmission.

During the first year of home monitoring, we included 320 patients (64\% male). Patient characteristics are shown in table 1 . A total of 196 patients (61\%) received oxygen therapy after discharge, ranging from 1 to $3 \mathrm{~L} \cdot \mathrm{min}^{-1}$. Mean \pm SD reduction in length of hospitalisation was $5.1 \pm 3.4$ days, and for patients with oxygen therapy $6.4 \pm 3.2$ days.

Reassessment at the emergency department was indicated in 39 (12\%) patients. Readmission to the ward was necessary in 23 (7\%) patients, which included 17 (74\%) males with mean \pm sD age $62 \pm 10$ years. No fatalities occurred. The main reasons for readmission were hypoxaemia $(n=15)$ and pulmonary embolism $(n=5)$. Underlying comorbidities in readmitted patients were chronic heart disease $(n=11 ; 48 \%)$, chronic lung disease $(n=11 ; 48 \%)$, hypertension $(n=10 ; 43 \%)$, diabetes mellitus $(n=4 ; 17 \%)$, obesity $(n=3 ; 13 \%)$, fever at time of discharge $(n=2 ; 9 \%)$, and immunocompromised state $(n=1 ; 4 \%) .16(70 \%)$ readmitted

Shareable abstract (@ERSpublications)

Home monitoring reduces hospital stay of patients with COVID-19, which increases regular healthcare capacity https://bit.ly/3CdFp1z

Cite this article as: Grutters LA, Majoor KI, Pol-Mattern ESK, et al. Home-monitoring reduces hospital stay for COVID-19 patients. Eur Respir J 2021; 58: 2101871 [DOI: 10.1183/13993003.01871-2021]. 
TABLE 1 Patient characteristics of the COVID-19 home monitoring cohort

Total cohort $(n=320)$ Readmitted patients $(n=23)$

\begin{tabular}{|c|c|c|}
\hline \multicolumn{3}{|l|}{ Gender } \\
\hline Male & $206(64)$ & $17(74)$ \\
\hline Age, years & $56 \pm 12$ & $62 \pm 10$ \\
\hline \multicolumn{3}{|l|}{ COVID-19 diagnosis based on } \\
\hline PCR & $318(99)$ & $23(100)$ \\
\hline Computed tomography & $1(0.3)$ & 0 \\
\hline Chest radiograph & $1(0.3)$ & 0 \\
\hline \multicolumn{3}{|l|}{ Underlying comorbidities } \\
\hline Diabetes mellitus & $39(12)$ & $4(17)$ \\
\hline Hypertension & $89(28)$ & $10(43)$ \\
\hline Obesity $\left(\mathrm{BMI}>30 \mathrm{~kg} \cdot \mathrm{m}^{-2}\right)$ & $100(31)$ & $3(13)$ \\
\hline Chronic pulmonary disease & $90(28)$ & $11(48)$ \\
\hline Chronic heart disease & $54(17)$ & $11(48)$ \\
\hline Immunocompromised state & $10(3)$ & $1(4)$ \\
\hline Admission to intensive care unit & $54(17)$ & $1(4)$ \\
\hline Length of stay on intensive care unit, days & $9 \pm 8.5$ & 5 \\
\hline \multicolumn{3}{|l|}{ Oxygen therapy during hospitalisation } \\
\hline Nasal oxygen or non-rebreather mask & $260(82)$ & $21(91)$ \\
\hline Non-invasive ventilation or nasal high flow oxygen therapy & $45(14)$ & $2(9)$ \\
\hline Intubation & $11(3.4)$ & 0 \\
\hline Duration of disease on admission to home monitoring, days & $18 \pm 9.1$ & $15.6 \pm 10.5$ \\
\hline Length of hospitalisation, days & $8.6 \pm 6.6$ & $6.8 \pm 5.2$ \\
\hline Fever at time of discharge & $7(2)$ & $2(9)$ \\
\hline \multicolumn{3}{|l|}{ Oxygen therapy when discharged } \\
\hline Yes & $196(61)$ & $16(70)$ \\
\hline No & $124(39)$ & $7(30)$ \\
\hline \multicolumn{3}{|l|}{ Total reduction in length of hospitalisation, days } \\
\hline In need for oxygen therapy $(n=189)^{\#}$ & 1200 & \\
\hline No need for oxygen therapy $(n=76)^{4}$ & 141.5 & \\
\hline Mean reduction in length of stay, days $(n=265)$ & $5.1 \pm 3.4$ & \\
\hline Length of home monitoring, days & $11.7 \pm 5.4$ & \\
\hline Number of telephone contacts & $5.7 \pm 3.0$ & \\
\hline
\end{tabular}

patients used oxygen at home, which was not a risk factor $(p=0.51)$. Chronic heart disease $(p=0.00)$ and obesity $(\mathrm{p}=0.02$ ) were risk factors for readmission. The other reported underlying comorbidities and fever at time of discharge were not identified as significant risk factors.

Home monitoring was rated as user-friendly by $93 \%$. It took $73 \%$ less than 10 min daily to take the measurements and complete the relevant details in the app. In, respectively, $14 \%$ and $83 \%$ of cases it was mostly or always clear what to do when low oxygen saturation was measured. 98\% would recommend home monitoring to acquaintances. The limitation of our study is the absence of a control group; therefore, it is difficult to draw conclusions regarding the generalisability of our outcomes.

In summary, while DiRIKGIL et al. [1] focused on a monitoring programme of suspected COVID-19 patients presenting to the emergency department, we focus on home monitoring of severe COVID-19 patients after discharge from the hospital. In addition to DiRIKGIL et al. [1], who showed home-monitoring reduced short stay admissions, we confirm that home monitoring reduces hospital stay of hospitalised COVID-19 patients in the largest patient cohort to date. This increases capacity for regular healthcare. Compared to our pilot project ( $\mathrm{n}=33$ ), we confirm the average reduction is hospitalisation of $5.1 \pm 3.4$ days [2]. Our readmission rate of $7 \%$ is comparable to $9 \%$ reported by DiRIKGIL et al. [1] and $12 \%$ reported by VAn Herwerden et al. [5]. Chronic heart disease and obesity were risk factors for readmission. In our cohort, fever at discharge was not a risk factor for readmission, in contrast to VAN HeRwerden et al. [5] $(p=0.01)$. Another important confirmation is the high patient satisfaction which is comparable to the literature. This highlights that home monitoring positively contributes to patient's health. 
To conclude, we confirm that home monitoring reduces hospital stay of COVID-19 patients in the largest patient cohort to date.

\section{Laura Agnes Grutters $\oplus^{1,4}$, Kalle Iskander Majoor ${ }^{1,4}$, Eline Solange Kirsty Pol-Mattern ${ }^{1}$, Johannes Anthonie Hardeman ${ }^{1}$, Christiaan Franciscus Pieter van Swol ${ }^{2}$ and Adriane Dore Marie Vorselaars ${ }^{1,3}$}

${ }^{1}$ Interstitial Lung Diseases Center of Excellence, Dept of Pulmonology, St Antonius Hospital, Nieuwegein, The Netherlands. ${ }^{2}$ Dept of Medical Physics, St. Antonius Hospital, Nieuwegein, The Netherlands. ${ }^{3}$ Division of Heart and Lungs, Utrecht University Medical Centre, Utrecht, The Netherlands. ${ }^{4}$ These authors contributed equally to the work.

Corresponding author: Adriane Dore Marie Vorselaars (a.vorselaars@antoniusziekenhuis.nl)

Acknowledgement: We would like to thank F. de Winter and the home monitoring team of the St Antonius Hospital for their commitment to this study.

Author contributions: All authors made substantial contributions to the conception or design of the work, the analysis, and interpretation of data. L.A. Grutters, K.I. Majoor and A.D.M. Vorselaars drafted the work. Critical revision was done by A.D.M. Vorselaars, E.S.K. Pol-Mattern, J.A. Hardeman and C.F.P. van Swol. All authors approved the final version to be published. All authors agreed to be accountable for all aspects of the work in ensuring that questions related to the accuracy or integrity of any part of the work are appropriately investigated and resolved.

Conflict of interest: None declared.

\section{References}

1 Dirikgil E, Roos R, Groeneveld $\mathrm{GH}$, et al. Home-monitoring reduced short stay admissions in suspected COVID-19 patients: COVID-box project. Eur Respir J 2021; 58: 2100636.

2 Grutters LA, Majoor KI, Mattern ESK, et al. Home telemonitoring makes early hospital discharge of COVID-19 patients possible. J Am Med Informatics Assoc 2020; 27: 1825-1827.

3 Annis T, Pleasants S, Hultman G, et al. Rapid implementation of a COVID-19 remote patient monitoring program. J Am Med Informatics Assoc 2020; 27: 1326-1330.

4 Hollander JE, Carr BG. Virtually perfect? Telemedicine for Covid-19. N Engl J Med 2020; 382: 1677-1679.

5 Van Herwerden MC, Van Steenkiste J, El Moussaoui R, et al. Thuisbehandeling van covid-19-patiënten met zuurstof en telemonitoring [Home telemonitoring and oxygen therapy in COVID-19 patients: safety, patient satisfaction, and cost-effectiveness]. Ned Tijdschr Geneeskd 2021; 165: D5740.

6 O'Carroll O, MacCann R, O'Reilly A, et al. Remote monitoring of oxygen saturation in individuals with COVID-19 pneumonia. Eur Respir J 2020; 56: 2001492.

7 Williamson EJ, Walker AJ, Bhaskaran $\mathrm{K}$, et al. Factors associated with COVID-19-related death using OpenSAFELY. Nature 2020; 584: 430-436.

8 Meuwissen L, de Bakker D. CQ-index huisartsenzorg: meetinstrumentontwikkeling. Utrecht, NIVEL, 2008. Available from: www.nivel.nl/sites/default/files/bestanden/CQ-index-huisartsenzorg-Meetinstrumentontwikkeling-v02-2008.pdf 